\title{
OUR JOURNAL, FROM DEBUT TO MODERNISM
}

Our journal, now at its $13^{\text {th }}$ annual edition, appeared out of the need of the group of teachers and economists (which was only at its beginnings during the 90s of the previous century) to make the results of their scientific studies and research well known. The published material was selected only after most of the articles were presented and discussed at scientific conferences organized at the level of the department or of the faculty. In fact, the precursors of the actual journal were the volumes containing the papers presented at the scientific conferences organized by the department specialized in economy in 1991 and 1995. Starting with 1996 the actual journal has been issued, with the title "Scientific Studies and Research", within the Economic Sciences series, with an editorial board coordinated by PhD Professor Vasile Pătruț and a number of scientific reviewers starting with number 5/2001. Among the most constant collaborators we mention Associate Professor Roxana Mironescu and Associate Professor Marcela Danu, who actually dealt with the editing of the journal, the former being in charge of the first seven issues of the journal, and, the latter, of four volumes of the journal.

We mention that, starting with number 3/1999, our journal has been accredited by CNCSIS and included in category $D$.

The areas of study covered by our collaborators are various and of imperative actuality, referring to the fields of economy and business management, as well as to the ones related to the economic education.

Having in view the relatively limited experience of our collaborators, the materials presented in the journal were only occasionally the results of advanced research. In many cases, the research was restricted only to bibliographic readings, sometimes followed by a direct research on the real facts, but without accomplishing what we call the "laboratory" research. Under the circumstances, by means of the published articles we only rarely promoted solutions, recommendations, and, most important, new interpretations or theories, as well as research instruments and methods. The published articles were most often some means of "popularizing" the well-known truths, and so they were not really "scientific" articles which presented new realities resulting from the undertaken research.

After a period of quantitative accumulations (see table no.1), on the basis of the gained experience of our collaborators in the field of research, among whom the majority are doctors and doctoral candidates in economic sciences, we consider that now is the time for a qualitative shift, so as to be able to ensure the necessary conditions for the upgrading of our journal by CNCSIS to superior categories in the hierarchy of scientific journals.

Among the objectives we have to set, with this aim, we mention the following:

- the improvement of the scientific quality of the published articles, thus promoting the articles which are truly valuable, and thus recommended for publication by the editing board, who must accomplish its task with all the requisite responsibility;

- the presentation and discussion of the materials which are due for publication on the occasion of the faculty's scientific sessions, hence renewing the practice of organizing annual editions of the conference "Contemporary Economy and the Romanian Realities", or the organizing of scientific symposia on certain themes like the ones organized by the Department of Accounting and Finances, such as "Provocations for the Accounting Profession in the Future GlobalInformational Society" - 2006, "Accounting and the Information and Communication Technologies" - January 2007, "Accounting within the Romanian Contemporariness" November 2007;

- the reorganization of the editorial board of the journal by attracting some acknowledged personalities in the domain of the economic sciences, academicians, or university professors from Romania and especially from foreign countries, etc.;

- the restructuring of the board of scientific reviewers so as to include renowned personalities within the same area of study; 
- ensuring a broader accessibility of our journal within and towards the group of experts at the national and international level, by performing exchanges with publications from other countries, or by promoting the articles of collaborators from other universities in our country or abroad;

- $\quad$ the publishing of the journal both in Romanian and in an international language;

The achievement of all these objectives and of others of the same kind should ensure the continuation of the journal's publishing, as well as the heightening of its prestige, and these are some targets of the future editorial board.

\section{References:}

[1] Zaiț, Dumitru, Spalanzani Alain, Research in Economy and Management, Editura Economică, Bucureşti, 2006

[2] ***, Studies and Scientific Researches, Edition: Economics, the collection from 1991 to 2008

[3] *** www.cncsis.ro

Vasile Pătruț,Managing Editor

Professor PhD, University of Bacău 


\begin{tabular}{|c|c|c|c|c|c|c|c|c|c|}
\hline No. & $\begin{array}{c}\text { Year of } \\
\text { Publication }\end{array}$ & Title of article & ISSN & Editor & Editorial Board & $\begin{array}{l}\text { Scientific } \\
\text { Reviewers }\end{array}$ & $\begin{array}{l}\text { Number } \\
\text { of Pages }\end{array}$ & $\begin{array}{c}\text { Number } \\
\text { of } \\
\text { Articles }\end{array}$ & $\begin{array}{l}\text { Occasion of } \\
\text { Publication }\end{array}$ \\
\hline \multicolumn{10}{|c|}{ Precursors of the Journal } \\
\hline 1. & 1991 & $\begin{array}{l}\text { Studies and } \\
\text { Research I- } \\
\text { Economy- Law - } \\
\text { Philosophy - } \\
\text { History }\end{array}$ & - & $\begin{array}{l}\text { The University of } \\
\text { Bacau, The Faculty } \\
\text { of Letters and } \\
\text { Sciences } \\
\text { Section Tourism - } \\
\text { Services - Marketing }\end{array}$ & $\begin{array}{l}\text { Associate Professor I. } \\
\text { Cioban - scientific } \\
\text { secretary of the Faculty }\end{array}$ & - & 153 & 32 & $\begin{array}{l}\text { The Session of Scientific } \\
\text { Communications of the } \\
\text { Department of Socio- } \\
\text { Humanistic Sciences and } \\
\text { Philology, I edition }\end{array}$ \\
\hline 2. & 1995 & $\begin{array}{l}\text { Studies and } \\
\text { Research in } \\
\text { Economy and } \\
\text { Law }\end{array}$ & - & $\begin{array}{l}\text { The University of } \\
\text { Bacau, The Faculty } \\
\text { of Economic and } \\
\text { Social Sciences }\end{array}$ & $\begin{array}{l}\text { PhD Professor C. Gh. } \\
\text { Marinescu } \\
\text { PhD Associate Professor } \\
\text { Vasile Pătrut } \\
\text { PhD Associate Professor } \\
\text { P. Rusu } \\
\text { University Lecturer, PhD } \\
\text { candidate L. Saradici } \\
\text { Lecturer, PhD candidate } \\
\text { R. Mironescu } \\
\end{array}$ & - & 193 & 27 & $\begin{array}{l}\text { The Session of Scientific } \\
\text { Communications of the } \\
\text { Department of Economic } \\
\text { and Social Sciences, II } \\
\text { edition, 12-13 May } 1995\end{array}$ \\
\hline \multicolumn{10}{|c|}{ “Scientific Studies and Research” Journal, the Economic Sciences series } \\
\hline 3. & 1996 & No. $1 / 1996$ & $\begin{array}{l}1224- \\
9858\end{array}$ & $\begin{array}{l}\text { The University of } \\
\text { Bacau, The Faculty } \\
\text { of Sciences, } \\
\text { Economic Sciences } \\
\text { Series }\end{array}$ & $\begin{array}{l}\text { PhD Professor Vasile } \\
\text { Pătruț } \\
\text { PhD Associate Professor } \\
\text { L. Saradici } \\
\text { PhD Associate Professor } \\
\text { R. Pintilie } \\
\text { Lecturer, PhD candidate } \\
\text { R. Mironescu }\end{array}$ & - & 104 & 26 & $\begin{array}{l}\text { The conference } \\
\text { organized to celebrate } \\
\text { the } 35 \text { - Years Jubilee of } \\
\text { Academic Education in } \\
\text { Bacau }\end{array}$ \\
\hline 4. & 1998 & No. 2/1998 & $\begin{array}{l}1224- \\
9858\end{array}$ & $\begin{array}{l}\text { The University of } \\
\text { Bacau, The Faculty } \\
\text { of Letters and } \\
\text { Sciences } \\
\text { Department of } \\
\text { Economic Sciences }\end{array}$ & $\begin{array}{l}\text { PhD Professor Vasile } \\
\text { Pătruț } \\
\text { PhD Professor R. Pintilie } \\
\text { PhD Associate Professor } \\
\text { L. Saradici } \\
\text { Lecturer, PhD candidate } \\
\text { R. Mironescu } \\
\end{array}$ & - & 78 & 18 & $\begin{array}{l}\text { The Session of Scientific } \\
\text { Communications of the } \\
\text { Department of Economic } \\
\text { Sciences }\end{array}$ \\
\hline 5. & 1999 & No. 3/1999 & $\begin{array}{l}1224- \\
9858\end{array}$ & $\begin{array}{l}\text { The University of } \\
\text { Bacau, The Faculty } \\
\text { of Letters and } \\
\text { Sciences } \\
\text { Department of }\end{array}$ & $\begin{array}{l}\text { PhD Professor Vasile } \\
\text { Pătrut - coordinator } \\
\text { PhD Professor R. Pintilie } \\
\text { PhD Associate Professor } \\
\text { L. Saradici }\end{array}$ & - & 141 & 19 & $\begin{array}{l}\text { The Annual Session of } \\
\text { Scientific } \\
\text { Communications of the } \\
\text { Department of Economic } \\
\text { Sciences }\end{array}$ \\
\hline
\end{tabular}




\begin{tabular}{|c|c|c|c|c|c|c|c|c|c|}
\hline & & & & Economic Sciences & $\begin{array}{l}\text { Lecturer, } \mathrm{PhD} \text { candidate } \\
\text { R. Mironescu }\end{array}$ & & & & \\
\hline 6. & 2000 & No. $4 / 2000$ & $\begin{array}{l}1224- \\
9858\end{array}$ & $\begin{array}{l}\text { The University of } \\
\text { Bacau, The Faculty } \\
\text { of Letters and } \\
\text { Sciences } \\
\text { Department of } \\
\text { Economic Sciences }\end{array}$ & $\begin{array}{l}\text { PhD Professor Vasile } \\
\text { Pătrut- coordinator } \\
\text { PhD Professor R. Pintilie } \\
\text { PhD Associate Professor } \\
\text { L. Saradici } \\
\text { Lecturer, PhD candidate } \\
\text { R. Mironescu } \\
\end{array}$ & - & 155 & 24 & $\begin{array}{l}\text { The conference } \\
\text { organized to celebrate } \\
\text { the } 10 \text {-Years Jubilee of } \\
\text { Academic Economic } \\
\text { Education at the } \\
\text { University of Bacau }\end{array}$ \\
\hline 7. & 2001 & No. $5 / 2001$ & $\begin{array}{l}1224- \\
9858\end{array}$ & $\begin{array}{l}\text { The University of } \\
\text { Bacau, The Faculty } \\
\text { of Letters and } \\
\text { Sciences } \\
\text { Department of } \\
\text { Economic Sciences }\end{array}$ & $\begin{array}{l}\text { PhD Professor Vasile } \\
\text { Pătruț- coordinator } \\
\text { PhD Professor R. Pintilie } \\
\text { PhD Associate Professor } \\
\text { L. Saradici } \\
\text { Lecturer, PhD candidate } \\
\text { R. Mironescu }\end{array}$ & $\begin{array}{l}\text { PhD Professor I. Cătoiu - } \\
\text { AES Bucureşti } \\
\text { PhD Professor V. Nechita } \\
\text { - Univ. "Al. I. Cuza" Iaşi } \\
\text { PhD Professor R. Petriş - } \\
\text { Univ. "Al. I. Cuza" Iaşi } \\
\text { PhD Associate Professor } \\
\text { Gh. Epuran - Univ. Of } \\
\text { Bacău }\end{array}$ & 116 & 19 & $\begin{array}{l}\text { The Annual Session of } \\
\text { Scientific } \\
\text { Communications of the } \\
\text { Department of Economic } \\
\text { Sciences }\end{array}$ \\
\hline 8. & 2001 & No. $6 / 2001$ & $\begin{array}{l}1224- \\
9858\end{array}$ & $\begin{array}{l}\text { The University of } \\
\text { Bacau, The Faculty } \\
\text { of Letters and } \\
\text { Sciences } \\
\text { Department of } \\
\text { Economic Sciences }\end{array}$ & $\begin{array}{l}\text { PhD Professor Vasile } \\
\text { Pătruț- coordinator } \\
\text { PhD Professor R. Pintilie } \\
\text { PhD Associate Professor } \\
\text { L. Saradici } \\
\text { Lecturer, PhD candidate } \\
\text { R. Mironescu }\end{array}$ & $\begin{array}{l}\text { PhD Professor I. Cătoiu - } \\
\text { AES Bucureşti } \\
\text { PhD Professor V. Nechita } \\
\text { - Univ. "Al. I. Cuza" Iaşi } \\
\text { PhD Professor R. Petriş - } \\
\text { Univ. “Al. I. Cuza” Iaşi } \\
\text { PhD Associate Professor } \\
\text { Gh. Epuran - Univ. Of } \\
\text { Bacău }\end{array}$ & 94 & 17 & $\begin{array}{l}\text { The Annual Session of } \\
\text { Scientific } \\
\text { Communications of the } \\
\text { Department of Economic } \\
\text { Sciences }\end{array}$ \\
\hline 9. & 2002 & No. $7 / 2002$ & $\begin{array}{l}1224- \\
9858\end{array}$ & $\begin{array}{l}\text { The University of } \\
\text { Bacau, The Faculty } \\
\text { of Letters and } \\
\text { Sciences } \\
\text { Department of } \\
\text { Economic Sciences }\end{array}$ & $\begin{array}{l}\text { PhD Professor Vasile } \\
\text { Pătrut - managing editor } \\
\text { PhD Professor P. Rusu } \\
\text { PhD Professor L. } \\
\text { Saradici } \\
\text { PhD Lecturer R. } \\
\text { Mironescu - chief editor, } \\
\text { editor in charge of the } \\
\text { issue }\end{array}$ & $\begin{array}{l}\text { PhD Professor I. Cătoiu - } \\
\text { AES Bucureşti } \\
\text { PhD Professor V. Nechita } \\
\text { - Univ. "Al. I. Cuza” Iaşi } \\
\text { PhD Professor R. Petriş - } \\
\text { Univ. “Al. I. Cuza" Iaşi } \\
\text { PhD Professor Gh. } \\
\text { Epuran - Univ. Of Bacău }\end{array}$ & 132 & 28 & $\begin{array}{l}\text { The conference } \\
\text { organized to celebrate } \\
\text { the } 10 \text {-Years Jubilee of } \\
\text { Academic Economic } \\
\text { Education at the } \\
\text { University of Bacau }\end{array}$ \\
\hline 10. & 2003 & No. $8 / 2003$ & $\begin{array}{l}1224- \\
9858\end{array}$ & $\begin{array}{l}\text { The University of } \\
\text { Bacau, The Faculty } \\
\text { of Sciences } \\
\text { Department of } \\
\text { Economic Sciences }\end{array}$ & $\begin{array}{l}\text { PhD Professor Vasile } \\
\text { Pătrut - managing editor } \\
\text { PhD Professor L. } \\
\text { Saradici } \\
\text { PhD Associate Professor } \\
\text { R. Mironescu } \\
\text { PhD Associate Professor } \\
\text { M.C. Danu - chief editor, }\end{array}$ & $\begin{array}{l}\text { PhD Professor I. Cătoiu - } \\
\text { AES Bucureşti } \\
\text { PhD Professor V. Nechita } \\
\text { - Univ. “Al. I. Cuza” Iaşi } \\
\text { PhD Professor R. Petriş - } \\
\text { Univ. "Al. I. Cuza” Iaşi } \\
\text { PhD Professor V. } \\
\text { Răileanu - AES }\end{array}$ & 149 & 29 & $\begin{array}{l}\text { The Annual Session of } \\
\text { Scientific } \\
\text { Communications of the } \\
\text { Department of Economic } \\
\text { Sciences }\end{array}$ \\
\hline
\end{tabular}




\begin{tabular}{|c|c|c|c|c|c|c|c|c|c|}
\hline & & & & & $\begin{array}{l}\text { editor in charge of the } \\
\text { issue }\end{array}$ & $\begin{array}{l}\text { Bucureşti } \\
\text { PhD Professor Gh. } \\
\text { Epuran - Univ. Of Bacău }\end{array}$ & & & \\
\hline 11. & 2004 & No. 9/2004 & $\begin{array}{l}1224- \\
9858\end{array}$ & $\begin{array}{l}\text { The University of } \\
\text { Bacau, The Faculty } \\
\text { of Sciences } \\
\text { Department of } \\
\text { Economic Sciences }\end{array}$ & $\begin{array}{l}\text { PhD Professor Vasile } \\
\text { Pătrut-- managing editor } \\
\text { PhD Professor L. } \\
\text { Saradici } \\
\text { PhD Associate Professor } \\
\text { M.C. Danu PhD } \\
\text { Associate Professor R. } \\
\text { Mironescu } \\
\text { - chief editor, editor in } \\
\text { charge of the issue }\end{array}$ & $\begin{array}{l}\text { PhD Professor I. Cătoiu - } \\
\text { AES Bucureşti } \\
\text { PhD Professor V. Nechita } \\
\text { - Univ. “Al. I. Cuza” Iaşi } \\
\text { PhD Professor V. } \\
\text { Răileanu - AES } \\
\text { Bucureşti } \\
\text { PhD Professor Gh. } \\
\text { Epuran - Univ. Of Bacău }\end{array}$ & 165 & 34 & $\begin{array}{l}\text { The Annual Session of } \\
\text { Scientific } \\
\text { Communications of the } \\
\text { Department of Economic } \\
\text { Sciences }\end{array}$ \\
\hline 12. & 2005 & No. $10 / 2005$ & $\begin{array}{l}1224- \\
9858\end{array}$ & $\begin{array}{l}\text { The University of } \\
\text { Bacau, The Faculty } \\
\text { of Economic } \\
\text { Sciences }\end{array}$ & $\begin{array}{l}\text { PhD Professor Vasile } \\
\text { Pătrut- managing editor } \\
\text { PhD Professor L. } \\
\text { Saradici } \\
\text { PhD Professor Gh. } \\
\text { Epuran } \\
\text { PhD Associate Professor } \\
\text { R. Mironescu } \\
\text { PhD Associate } \\
\text { Professor M.C. Danu } \\
\text { - chief editor, editor in } \\
\text { charge of the issue }\end{array}$ & $\begin{array}{l}\text { PhD Professor I. Cătoiu - } \\
\text { AES București } \\
\text { PhD Professor V. Nechita } \\
\text { - Univ. “Al. I. Cuza" Iaşi } \\
\text { PhD Professor V. } \\
\text { Răileanu - AES } \\
\text { București } \\
\text { PhD Professor I. Florea - } \\
\text { Univ. "Al. I. Cuza” Iaşi }\end{array}$ & 204 & 30 & $\begin{array}{l}\text { National Conference } \\
\text { with International } \\
\text { Participants } \\
\text { "Contemporary } \\
\text { Economy and Romanian } \\
\text { Realities", } 11-12 \\
\text { November } 2005 \text {, } \\
\text { dedicated to the } \\
\text { anniversary of } 15 \text { years } \\
\text { of academic education in } \\
\text { Bacau }\end{array}$ \\
\hline 13. & 2006 & No. $11 / 2006$ & $\begin{array}{l}1224- \\
9858\end{array}$ & $\begin{array}{l}\text { The University of } \\
\text { Bacau, The Faculty } \\
\text { of Economic } \\
\text { Sciences }\end{array}$ & $\begin{array}{l}\text { PhD Professor Vasile } \\
\text { Pătrut-- managing editor } \\
\text { PhD Professor L. } \\
\text { Saradici } \\
\text { PhD Professor Gh. } \\
\text { Epuran } \\
\text { PhD Associate Professor } \\
\text { R. Mironescu } \\
\text { PhD Associate } \\
\text { Professor M.C. Danu } \\
\text { - chief editor, editor in } \\
\text { charge of the issue }\end{array}$ & $\begin{array}{l}\text { PhD Professor I. Cătoiu - } \\
\text { AES Bucureşti } \\
\text { PhD Professor V. Nechita } \\
\text { - Univ. "Al. I. Cuza" Iaşi } \\
\text { PhD Professor V. } \\
\text { Răileanu - AES } \\
\text { București } \\
\text { PhD Professor I. Florea - } \\
\text { Univ. "Al. I. Cuza" Iaşi }\end{array}$ & 180 & 35 & $\begin{array}{l}\text { National Conference } \\
\text { with International } \\
\text { Participants } \\
\text { "Contemporary } \\
\text { Economy and Romanian } \\
\text { Realities", II }{ }^{\text {nd }} \text { Edition }\end{array}$ \\
\hline 14. & 2007 & $\begin{array}{l}\text { No. 12/2007 } \\
\text { (in English) }\end{array}$ & $\begin{array}{l}1224- \\
9858\end{array}$ & $\begin{array}{l}\text { The University of } \\
\text { Bacau, The Faculty } \\
\text { of Economic } \\
\text { Sciences }\end{array}$ & $\begin{array}{l}\text { PhD Professor Vasile } \\
\text { Pătruț- managing editor } \\
\text { PhD Professor L. } \\
\text { Saradici } \\
\text { PhD Professor Gh. } \\
\text { Epuran } \\
\text { PhD Associate }\end{array}$ & $\begin{array}{l}\text { PhD Professor I. Cătoiu - } \\
\text { AES Bucureşti } \\
\text { PhD Professor V. Nechita } \\
\text { - Univ. "Al. I. Cuza" Iaşi } \\
\text { PhD Professor V. } \\
\text { Răileanu - AES } \\
\text { Bucureşti }\end{array}$ & 131 & 25 & $\begin{array}{l}\text { National Scientific } \\
\text { Symposium "Accounting } \\
\text { within the Romanian } \\
\text { Contemporariness"- } \\
\text { November } 2007 \text {, } \\
\text { dedicated to the } \\
\text { anniversary of } 10 \text { years }\end{array}$ \\
\hline
\end{tabular}




\begin{tabular}{|c|c|c|c|c|c|c|c|c|c|}
\hline & & & & & $\begin{array}{l}\text { Professor M.C. Danu } \\
\text { PhD Associate Professor } \\
\text { R. Mironescu } \\
\text { - chief editor, editor in } \\
\text { charge of the issue }\end{array}$ & $\begin{array}{l}\text { PhD Professor R. Petriş - } \\
\text { Univ. "Ştefan cel Mare", } \\
\text { Suceava }\end{array}$ & & & $\begin{array}{l}\text { of academic education } \\
\text { specialized in accounting } \\
\text { in Bacău }\end{array}$ \\
\hline 15. & 2008 & $\begin{array}{l}\text { No. } 13 / 2008 \\
\text { (bilingual edition) }\end{array}$ & $\begin{array}{l}1224- \\
9858\end{array}$ & $\begin{array}{l}\text { The University of } \\
\text { Bacau, The Faculty } \\
\text { of Economic } \\
\text { Sciences }\end{array}$ & $\begin{array}{l}\text { PhD Professor Vasile } \\
\text { Pătruț- managing editor } \\
\text { PhD Professor L. } \\
\text { Saradici } \\
\text { PhD Professor Gh. } \\
\text { Epuran } \\
\text { PhD Associate } \\
\text { Professor M.C. Danu } \\
\text { - chief editor, editor in } \\
\text { charge of the issue } \\
\text { PhD Associate Professor } \\
\text { R. Mironescu }\end{array}$ & $\begin{array}{l}\text { PhD Professor I. Cătoiu - } \\
\text { AES Bucureşti } \\
\text { PhD Professor V. Nechita } \\
\text { - Univ. "Al. I. Cuza" Iaşi } \\
\text { PhD Professor V. } \\
\text { Răileanu - AES } \\
\text { Bucureşti } \\
\text { PhD Professor R. Petriş - } \\
\text { Univ. "Ştefan cel Mare", } \\
\text { Suceava }\end{array}$ & 127 & 22 & $\begin{array}{l}\text { National Conference } \\
\text { with International } \\
\text { Participants } \\
\text { "Contemporary } \\
\text { Economy and Romanian } \\
\text { Realities", III }{ }^{\text {nd }} \text { Edition }\end{array}$ \\
\hline
\end{tabular}

